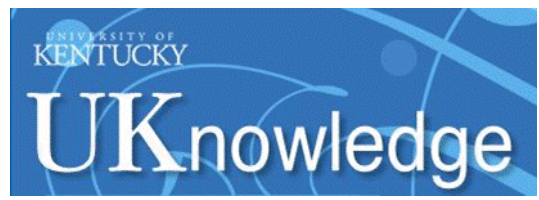

University of Kentucky

UKnowledge

\title{
Band Offsets at the Interface Between Crystalline and Amorphous Silicon from First Principles
}

\author{
Karol Jarolimek \\ University of Kentucky, karol.jarolimek@uky.edu \\ E. Hazrati \\ Radboud University, The Netherlands \\ R. A. de Groot \\ Radboud University, The Netherlands \\ D. A. de Wijs \\ Radboud University, The Netherlands
}

Follow this and additional works at: https://uknowledge.uky.edu/caer_facpub

Part of the Atomic, Molecular and Optical Physics Commons, and the Engineering Science and Materials Commons

Right click to open a feedback form in a new tab to let us know how this document benefits you.

\section{Repository Citation}

Jarolimek, Karol; Hazrati, E.; de Groot, R. A.; and de Wijs, D. A., "Band Offsets at the Interface Between Crystalline and Amorphous Silicon from First Principles" (2017). Center for Applied Energy Research Faculty and Staff Publications. 20.

https://uknowledge.uky.edu/caer_facpub/20

This Article is brought to you for free and open access by the Center for Applied Energy Research at UKnowledge. It has been accepted for inclusion in Center for Applied Energy Research Faculty and Staff Publications by an authorized administrator of UKnowledge. For more information, please contact UKnowledge@lsv.uky.edu. 


\title{
Band Offsets at the Interface Between Crystalline and Amorphous Silicon from First Principles
}

\author{
Digital Object Identifier (DOI) \\ https://doi.org/10.1103/PhysRevApplied.8.014026 \\ Notes/Citation Information \\ Published in Physical Review Applied, v. 8, issue 1, 014026, p. 1-6. \\ (C) 2017 American Physical Society
}

The copyright holder has granted the permission for posting the article here.

This article is available at UKnowledge: https://uknowledge.uky.edu/caer_facpub/20 


\title{
Band Offsets at the Interface between Crystalline and Amorphous Silicon from First Principles
}

\author{
K. Jarolimek, ${ }^{1, \dagger}$ E. Hazrati, ${ }^{1}$ R. A. de Groot, ${ }^{1,2}$ and G. A. de Wijs ${ }^{1, *}$ \\ ${ }^{1}$ Radboud University, Institute for Molecules and Materials, \\ Heyendaalseweg 135, 6525 AJ Nijmegen, The Netherlands \\ ${ }^{2}$ Zernike Institute for Advanced Materials, University of Groningen, \\ Nijenborgh 4, 9747 AG Groningen, The Netherlands
}

(Received 22 December 2016; revised manuscript received 10 May 2017; published 24 July 2017)

\begin{abstract}
The band offsets between crystalline and hydrogenated amorphous silicon $(a-\mathrm{Si}: \mathrm{H})$ are key parameters governing the charge transport in modern silicon heterojunction solar cells. They are an important input for macroscopic simulators that are used to further optimize the solar cell. Past experimental studies, using $\mathrm{x}$-ray photoelectron spectroscopy (XPS) and capacitance-voltage measurements, have yielded conflicting results on the band offset. Here, we present a computational study on the band offsets. It is based on atomistic models and density-functional theory (DFT). The amorphous part of the interface is obtained by relatively long DFT first-principles molecular-dynamics runs at an elevated temperature on 30 statistically independent samples. In order to obtain a realistic conduction-band position the electronic structure of the interface is calculated with a hybrid functional. We find a slight asymmetry in the band offsets, where the offset in the valence band $(0.29 \mathrm{eV})$ is larger than in the conduction band $(0.17 \mathrm{eV})$. Our results are in agreement with the latest XPS measurements that report a valence-band offset of $0.3 \mathrm{eV}$ [M. Liebhaber et al., Appl. Phys. Lett. 106, 031601 (2015)].
\end{abstract}

DOI: 10.1103/PhysRevApplied.8.014026

\section{INTRODUCTION}

Silicon heterojunction (SHJ) solar cells combine the high efficiency of $c$-Si wafer technology with the high throughput and low cost of hydrogenated amorphous silicon $(a-\mathrm{Si}: \mathrm{H})$ solar cells. The interface between crystalline and amorphous silicon lies at the heart of the SHJ solar cell. Since $a$-Si:H has a larger band gap than $c$-Si, band offsets are formed at the interface.

Experimentally, the band offsets can be determined with techniques such as photoelectron spectroscopy and capacitance-voltage measurements. The reported values, however, scatter in a broad range [1]. This can be due to different deposition conditions of the $a-\mathrm{Si}: \mathrm{H}$ layer or a misinterpretation of the experimental results. On average, it appears that the offset at the valence band is larger than at the conduction band (Ref. [1], p. 418, Refs. [2,3]).

Theoretical studies were mostly concerned with the atomic structure of the interface between $c$-Si and pure $a$-Si. Studies aimed either to obtain the interface energy (Ref. [4]) or to study the velocity of the crystallization of $a$-Si on $c$-Si substrates [5]. Some studies reported the electronic density of states, for the $c-\mathrm{Si}$ and $a-\mathrm{Si}: \mathrm{H}$ parts of the interface, but did not comment on the band offsets [6-8]. Santos et al. [9] investigated defects present at the interface and the corresponding electronic levels within the

\footnotetext{
*g.dewijs@science.ru.nl

${ }^{\dagger}$ Present address: University of Kentucky, Center for Applied Energy Research, 2540 Research Park Drive, Lexington, KY 40511, USA.
}

band gap. George et al. [10] modeled the electron spin resonance signal of defects at the interface. In terms of band offset calculations, we are aware of two studies that obtained the values from the respective bulk materials [11,12]. Peressi et al. [13] used a complete interface model, prepared with a combination of classical and first-principle molecular dynamics [13]. Their amorphous part, was, however, build from pure $a$-Si and often contained a high defect concentration making the interface semimetallic.

We present a calculation of band offsets that improves upon published studies in several aspects. The band offsets are calculated from an explicit interface model and not extracted from bulk properties only. There is a substantial (30) number of independent structural models that allows for reliable statistics. These models are prepared entirely from first-principles molecular dynamics (MD) with defect levels sufficiently low to determine the band edges. The electronic structure is described with hybrid functionals that give a better description of band gaps and conductionband offsets. We restrict ourselves to the technologically most relevant case of the (111) interface. Although (100) wafers are commonly used, surface texturing used to increase light absorption leads to the formation of pyramids with (111) facets (Ref. [14], p. 38).

This paper is organized as follows. Section II provides technical details on the calculations. The preparation of the structural models is described in Sec. III. In Sec. IV we discuss the electronic structure of the interface. Conclusions are presented in Sec. VI. 


\section{TECHNICAL DETAILS}

Calculations are performed on the level of density functional theory (DFT) with the Vienna ab initio simulation package (VASP) $[15,16]$. Electron-ion interactions are described using the projector augmented wave (PAW) method $[17,18]$.

We perform molecular-dynamics calculations with the Verlet algorithm. The canonical $N V T$ ensemble is simulated using the algorithm by Nosé [19]. We increase the mass of hydrogen to $10 \mathrm{amu}$, which allows us to use a slightly longer time step of $1.5 \mathrm{fs}$. During the whole MD run and the relaxation, we use the $\Gamma$ point for Brillouinzone sampling. The kinetic energy cutoff is set relatively low at $150 \mathrm{eV}$. This is made possible by using PAW potentials with larger core radii [20]. For $\mathrm{Si}$, the $s-, p$-, and $d$-partial wave radii are 2.2, 2.7, and 2.7 a.u., respectively. For $\mathrm{H}$, both $s$ - and $p$-partial wave radii are 1.3 a.u. The performance of the potentials is tested on bulk $c$-Si and the $\mathrm{SiH}_{4}$ molecule. The equilibrium $\mathrm{Si}-\mathrm{Si}$ and $\mathrm{Si}-\mathrm{H}$ bond lengths decrease by less than $0.01 \AA$, when using the less accurate potentials. The frequency of the TO mode in $c$-Si decreases by $1 \%$, while the frequency of the $\mathrm{Si}-\mathrm{H}$ stretching mode in $\mathrm{SiH}_{4}$ is lower by $5 \%$. Test runs on pure $a-\mathrm{Si}: \mathrm{H}$ give results similar to previous work [21,22]. The abovedescribed tests, as well as all dynamic calculations, are performed with the generalized gradient approximation (GGA) using the Perdew-Burke-Ernzerhof functional for solids (PBEsol) [23].

Although the GGA gives accurate structural properties, it is known to underestimate band gaps. In order to obtain realistic band gaps and offsets [24], all static calculations are performed with a hybrid functional. This type of functional includes a part of exact exchange from Hartree-Fock theory. We use the HSE06 hybrid functional $[25,26]$ with a screening parameter of $0.2 \AA^{-1}$. PBE potentials with a $250-\mathrm{eV}$ cutoff are used. The Brillouin zone is sampled with a $\Gamma$-centered $2 \times 2 \times 1$ mesh, while the Hartree-Fock kernel is evaluated only at $\Gamma$. The density of states is calculated with a Gaussian smearing with a width of $0.05 \mathrm{eV}$.

\section{PREPARATION OF THE STRUCTURE}

In order to simulate the interface, we construct a total of 30 simulation cells with dimensions of $15.35 \times 13.30 \times$ $36.00 \AA^{3}$. Periodic boundary conditions are applied in all three dimensions. The cells are divided into a crystalline and an amorphous part. The crystalline part consists of three double layers of $\mathrm{Si}$ atoms, centered within a 9.40- $\AA$ wide region (see Fig. 1).

The amorphous part contains $256 \mathrm{Si}$ atoms and $30 \mathrm{H}$ atoms, which leads to a $\mathrm{H}$ concentration of 10.5 at. $\%$ and mass density of $2.21 \mathrm{~g} / \mathrm{cm}^{3}$. These values are representative of device-quality bulk $a$-Si:H [27]. Initially, the atoms are placed randomly in the cell, followed by an annealing step at $1100 \mathrm{~K}$ for $135 \mathrm{ps}$, using DFT molecular dynamics. The optimum annealing temperature is determined by a series of tests on bulk $a-\mathrm{Si}: \mathrm{H}$ cells. Low temperatures did not result in sufficient movement of atoms and the system could be trapped in a high-energy local minimum. On the other hand, a too-high temperature could result in a liquidlike structure with many overcoordinated defects.

After the annealing step, a relaxation is performed that brings the system to the nearest local energy minimum. During the annealing and relaxation, atoms in the crystalline part are fixed and are not allowed to move. As a last step, we double the amount of $c$-Si in the simulation cell. The cell vector perpendicular to the interface increases from 36.00 to $45.40 \AA$.

In the following, we analyze the structure of the middle portion of the amorphous cell. Only atoms that are located more than $3.1 \AA$ from the nearest interface are considered. The mean $\mathrm{Si}-\mathrm{Si}$ and $\mathrm{Si}-\mathrm{H}$ bond lengths are 2.37 and $1.53 \AA$, respectively. These values compare well with diffraction measurements on bulk $a-\mathrm{Si}: \mathrm{H}$, that give 2.35 and $1.48 \AA$, respectively [28]. On average, we find $2.1 \mathrm{H}-\mathrm{H}$ bonds $\left(\mathrm{H}_{2}\right.$ molecules) per simulation cell. A more sensitive measure of strain in the amorphous network is the bond-angle distribution. To proceed, we define the following cutoff distances: $r_{\mathrm{Si}-\mathrm{Si}}=2.76 \AA$ and $r_{\mathrm{Si}-\mathrm{H}}=1.79 \AA$. The bond angles are calculated only between $\mathrm{Si}-\mathrm{Si}$ bonds, and $\mathrm{Si}-\mathrm{H}$ bonds are ignored. We obtain an average value of $108.9^{\circ}$, that is close to the bulk experimental value of $109.5^{\circ}$ [28]. The bond-angle RMS deviation can be inferred from the width of the TO peak, as measured by Raman spectroscopy. The experimental values of $8.7^{\circ}$ (Ref. [29]) and $9.3^{\circ}$ (Ref. [30]) are a bit smaller than the calculated value of $12.6^{\circ}$. This points to some additional strain in our models, although one has to keep in mind that the experiments are done on bulk $a-\mathrm{Si}: \mathrm{H}$. It is reasonable to assume that the amorphous network is more strained close to the interface with $c$-Si.

Another important property of the $a-\mathrm{Si}: \mathrm{H}$ model is the number of coordination defects. We find that the number of fivefold-coordinated $\mathrm{Si}$ atoms is higher than the number of threefold-coordinated ones (4.6 and 1.4 atoms per simulation

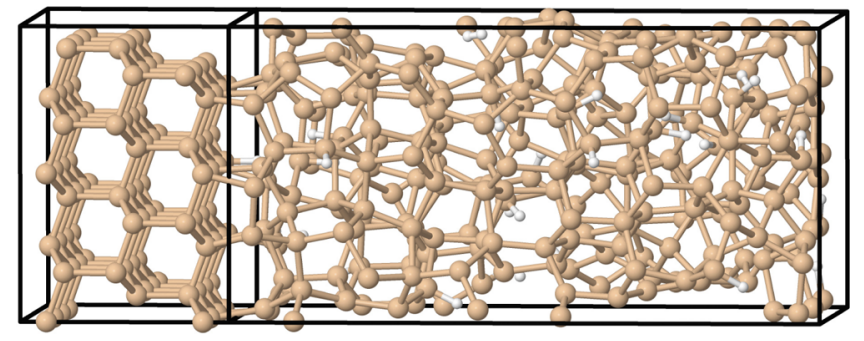

FIG. 1. One of the simulation cells used in the present study. The crystalline cell is terminated with two distinct (111) surfaces. $\mathrm{Si}$ and $\mathrm{H}$ atoms are drawn as brown and white spheres, respectively. 
cell, respectively). Tosolini et al. [8] reported an opposite trend and Santos et al. [9] found threefold-coordinated atoms but did not report on the fivefold-coordinated ones. We also find $\mathrm{H}$ atoms in a bridging position ( 0.2 atoms per simulation cell). Santos et al. [9] also investigated this defect but it was not reported by Tosolini et al. [8]. The number of other defects is less than 0.1 per simulation cell. The differences with Santos et al. and Tosolini et al. are possibly due to the different method used to prepare the structure: they used tight-binding MD, whereas we used DFT MD. The presence of the interface might lead to more fivefold-coordinated $\mathrm{Si}$ atoms. In our previous DFT MD study on pure $a-\mathrm{Si}: \mathrm{H}$, which had a similar $\mathrm{H}$ concentration, we obtained comparable numbers of threefold- and fivefold-coordinated Si atoms [22].

\section{ELECTRONIC STRUCTURE OF THE INTERFACE}

Because we have to average over 30 simulation cells, we need to align the single-particle energy levels in the different cells. We use the mean potential at the core of the $\mathrm{Si}$ atoms in the midsection of the crystalline part (2 layers, 32 atoms) for this purpose [31]. The potential at a particular atomic site is calculated with a unit test charge that has a radius of $0.989 \AA$.

In Fig. 2, we show the position-resolved density of states of the interface. There is a marked difference between the crystalline and amorphous parts of the interface. In the crystalline part, the six double layers of $\mathrm{Si}$ atoms can be easily identified as areas with a high density of states. The disorder in the amorphous part leads to a smeared-out DOS. Some ordering is visible close to the crystalline part. Evidently, the layered ordering is lost only gradually when moving away from the fixed crystalline part. The band gap is centered at around $85 \mathrm{eV}$ and has a dark blue color.

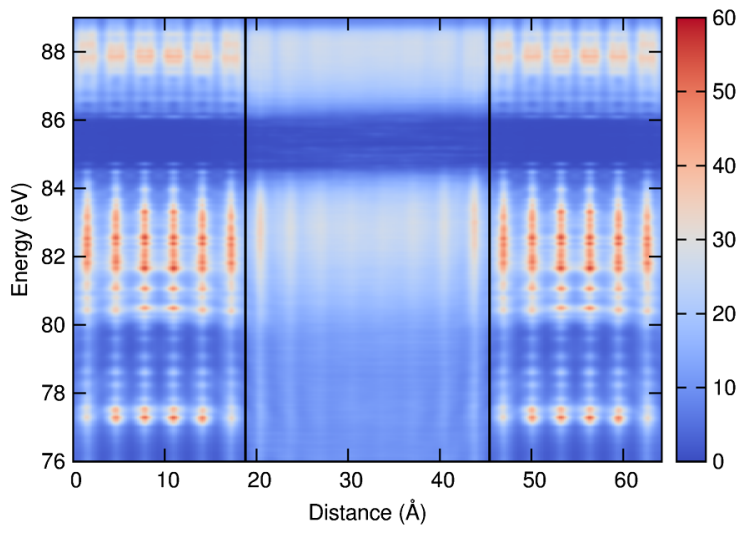

FIG. 2. Position-resolved DOS (in $10^{21} \mathrm{~cm}^{-3} \mathrm{eV}^{-1}$, HSE06) along the normal of the interface and obtained as an average over 30 cells. The crystalline part of the cell is shown twice in order to see both interfaces more clearly. Interfaces are marked by vertical lines. The zero of energy is the mean potential at the $\mathrm{Si}$ atom cores in the central part of the crystalline region.
In the following, we consider the DOS of the middle section of the amorphous part that is representative of bulk $a$-Si:H (see Fig. 3). We discard $3.1 \AA$ (width of one double layer) from both edges of the amorphous part. The determination of a band gap for amorphous semiconductors is somewhat ambiguous [32]. We use the definition by Tauc [33], which is the simplest one. In this model, the valence and conduction-band DOS follow a square-root dependence on energy (see red lines in Fig. 3). It is clear that the model is valid only for the middle range of DOS values. We choose an interval that spans from $30 \%$ to $80 \%$ of the maximum DOS value (at $28 \times 10^{21} \mathrm{~cm}^{-3} \mathrm{eV}^{-1}$ ). This allows us to find the energy ranges to fit the Tauc model to the calculated DOS (see Ref. [34] for details). Using a lower limit of $30 \%$ effectively means that we rely on the extended states to define the position of the band edge and that we suppress the effect of tail and defect states. This should also minimize the effect of stress in the structural models. After performing a least-square fit, we obtain a band gap of $1.60 \mathrm{eV}$ (HSE06), which is quite close to the experimental Tauc gap of $1.7 \mathrm{eV}$ [35]. We test the sensitivity of the obtained band edges on the number of cells used in the averaging. When using the first 10 or 20 cells the position of the band edges changed by less than $0.01 \mathrm{eV}$. Band offsets are affected by the same amount.

As a next step we need to obtain the position of the $c-\mathrm{Si}$ band edges. One might assume that one can do this by calculating the DOS of the crystalline part of the interface, in an analogous way as is done for the amorphous part. It turns out, however, that the band gap obtained in this way is overestimated. We argue that this is due to quantum confinement effects. To illustrate this we select one of the cells and calculate its band structure with the GGA functional (see Fig. 4). We chose a path in reciprocal space that is the same as for a primitive $c$-Si cell with two atoms [36]. The band structure of the interface cell is color coded

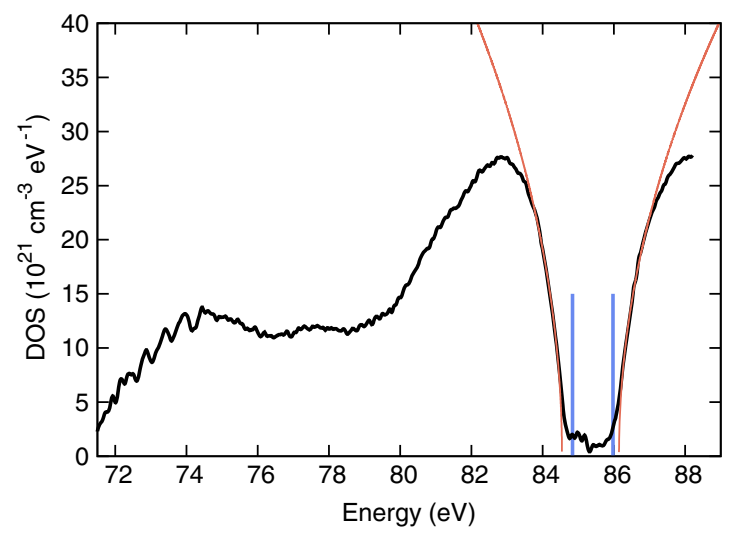

FIG. 3. HSE06 DOS of the amorphous part of the interface (black lines). The Tauc fit to the DOS is shown in red. The positions of band edges of $c$-Si are marked with blue vertical lines. The zero of energy is the mean potential at the $\mathrm{Si}$ atom cores in the central part of the crystalline region. 


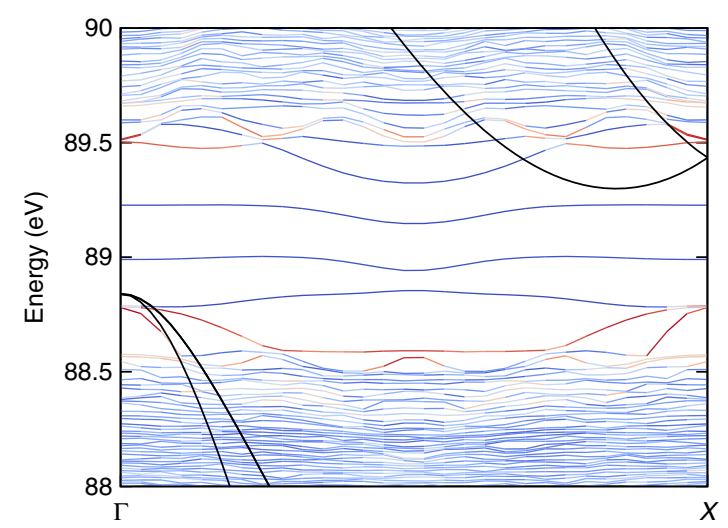

FIG. 4. PBEsol band structure of the interface between $c$-Si and $a$-Si:H compared to bulk $c$-Si (black lines). States marked with red (blue) color are on the crystalline (amorphous) side of the interface. The zero of energy is the mean potential at the Si atom cores in the central part of the crystalline region.

so that we can identify states (in red) that are localized in the crystalline part of the interface. We observe that the valence-band maximum is still at $\Gamma$ and the conductionband minimum is close to the $X$ point. In both cases, the bands are less dispersive than in bulk $c$-Si. The (PBEsol) band gap is larger $(0.69 \mathrm{eV})$ compared to bulk $c$-Si $(0.46 \mathrm{eV})$. When we remove the double layers of $\mathrm{Si}$ atoms $(9.40 \AA)$ from the crystalline part, we obtain an even larger band gap of $0.96 \mathrm{eV}$. Conversely, adding three double layers decreases the band gap to $0.58 \mathrm{eV}$. We note that we expect only weak quantum confinement and chargelocalization effects in the amorphous part, since the charge carriers in bulk $a-\mathrm{Si}: \mathrm{H}$ are localized to begin with [34]. In Ref. [34], using a similar amorphous model, confinement to a region of $16 \AA$ led to a band-gap deviation of $\sim 0.1 \mathrm{eV}$. In the present case, the confining potential is much shallower and the confinement region is much larger ( $\sim 25 \AA$ ), so the confinement effect should be negligible.

In order to circumvent the problem with quantum confinement in the $c$-Si part, we follow a different approach $[37,38]$. We calculate the band structure of $c$-Si with two atoms in the unit cell and obtain a band gap of $1.14 \mathrm{eV}$ (HSE06). The positions of the top of the valence band (at $\Gamma$ ) and the bottom of the conduction band (between $\Gamma$ and $X$ ) are again referenced to the potential at the core of the $\mathrm{Si}$ atoms [31]. These values are then combined with the DOS of $a-\mathrm{Si}: \mathrm{H}$ (see blue lines in Fig. 3). The band offsets are calculated as differences between the positions of the band edges in $c$-Si and $a-\mathrm{Si}: \mathrm{H}$. For the valence and conduction offset, we obtain 0.29 and $0.17 \mathrm{eV}$, respectively.

The Si-H bonds in the amorphous part form small dipoles. As our simulation cells contain about 30 of these, oriented in a randomized fashion, these dipoles can add up to a net nonvanishing dipole. In particular, this can give rise to a net dipole component in the direction perpendicular to the interfaces, which, because of the periodic boundary conditions, will also give rise to a net electric field in the crystalline slab. Of course, such fields are also present in reality, but there they average to 0 over larger length scales (as amorphous $a-\mathrm{Si}: \mathrm{H}$ is isotropic at macroscopic length scales). What remains are two opposite dipole layers at the interface, i.e., no net field is present inside the $c$-Si. In our case, the averaging over 30 independent (double) interface models mimics this: some cells have potential drops as large as $0.25 \mathrm{eV}$ going from the second to the fifth double layer (we have to discard the outer double layers, 1 and 6), but the averaged cell has a drop of $0.04 \mathrm{eV}$. We have a finite number of interface models, so averaging does not remove the field completely and we have to consider the average of both averaged surfaces. This yields an estimated inaccuracy due to the imperfect suppression of macroscopic fields of $0.02 \mathrm{eV}$. These fields are intrinsic to the procedure used to make the supercell structures, i.e., they cannot be avoided.

\section{DISCUSSION}

Our calculated valence-band offset $(0.29 \mathrm{eV})$ is in good agreement with the latest $\mathrm{X}$-ray photoelectron spectroscopy (XPS) measurement of $0.3 \mathrm{eV}$ [39]. Kleider performed a careful analysis of capacitance-voltage $(\mathrm{CV})$ measurements and reports a valence and conduction offset of 0.40 and $0.15 \mathrm{eV}$, respectively (Ref. [1], p. 405). Both are close to our values $(0.29$ and $0.17 \mathrm{eV})$. Kleider notes that some of the previous studies did not take into account the specific properties of $a-\mathrm{Si}: \mathrm{H}$ in their analysis. This could then explain the large spread in the reported values.

Previous theoretical studies used models of bulk $c$-Si and $a$-Si:H to calculate band offsets. Our results for the valence offset fall in between the reported values. Allan et al. used a $\sim 4000$ atom model of $a-\mathrm{Si}: \mathrm{H}$ and in the tight-binding approximation obtained a valence offset of $0.36 \mathrm{eV}$. The $\mathrm{H}$ concentration was 8 at.\%, close to our value, but the mass density was not reported. It is likely that the final value is, however, larger since the band edge states used are somewhat localized (band tails). This is corroborated by the fact that the reported band gap of $a-\mathrm{Si}: \mathrm{H}$ is too small $(1.36 \mathrm{eV})$ [11]. Van de Walle used the so-called "model-solid" theory to obtain a valence-band offset of $0.2 \mathrm{eV}$ [12]. Van de Walle also derived relations between the offsets and $a-\mathrm{Si}: \mathrm{H}$ density and $\mathrm{H}$ concentration. The $a-\mathrm{Si}: \mathrm{H}$ model has a similar $\mathrm{H}$ concentration (11 at.\%) as our models, but the density is higher (set to the $c$-Si value). When we substitute our density, to make a more fair comparison, the valence offset reduces to $0.07 \mathrm{eV}$. It is difficult to pinpoint the origin of the discrepancies between our results and the older studies. The fact that the interface is not considered directly might play a role, together with differences in $a-\mathrm{Si}: \mathrm{H}$ structure preparation and density. In our view, the most likely explanation is that the methods to locate the band edges in $a-\mathrm{Si}: \mathrm{H}$ are different. When small cells or a small number of cells are used, it is difficult to distinguish between extended and tail states. 
In terms of SHJ solar-cell performance there seems to be an optimum value for the valence-band offset. Several studies simulated the performance of $n$-type wafer-based SHJ cells by solving the Poisson and charge carrier continuity equations (see Ref. [40], and references therein). The studies agree that a valence offset larger than $0.5 \mathrm{eV}$ leads to a sharp decrease in solar-cell efficiency. This is caused by the accumulation and subsequent recombination of holes on the $c$-Si side of the $c$-Si/a-Si:H interface. Shen et al. put the optimum offset at $0.45 \mathrm{eV}$ [40].

\section{CONCLUSIONS}

We have prepared an atomistic model of the interface between crystalline and amorphous silicon. The amorphous part is hydrogenated and is thus relevant for technological applications such as silicon heterojunction solar cells. In order to obtain reliable results we average the calculated quantities over 30 statistically independent simulations cell. Atomic models are prepared with molecular dynamics, where forces are computed with density functional theory. This should give an accurate description of the interface region that contains a large number of strained bonds.

The electronic structures of the particular simulation cells are aligned at the mean potential at the $\mathrm{Si}$ atom cores in the crystalline part of the interface. We attempt to extract the band offsets directly from the position-resolved density of states. This is possible in the amorphous part but not in the crystalline part of the interface due to quantum confinement effects. To resolve this issue, we use band edges from a bulk $c$-Si calculation and perform again an alignment at the core potential of Si atoms. We obtain a valence and conduction-band offset of 0.29 and $0.17 \mathrm{eV}$. This is in good agreement with recent XPS and $C V$ measurements $[1,39]$.

\section{ACKNOWLEDGMENTS}

We thank Professor Kresse for the special, extra-soft PAW data sets. This work is supported by the Technology Foundation STW within the framework of the Fundamentals and Applications of Silicon Heterojunctions (FLASH) perspective program (Project Code 12165). We thank SURFsara for the support in using the Lisa Compute Cluster. The work of R. A. G. is part of the research program of the Foundation for Fundamental Research on Matter (FOM). Both SURFsara and FOM are financially supported by the Netherlands Organization for Scientific Research (NWO).

[1] J.-P. Kleider, Band Lineup Theories and the Determination of Band Offsets from Electrical Measurements in Physics and Technology of Amorphous-Crystalline Heterostructure Silicon Solar Cells, edited by W. G. J. H. M. van Sark, L. Korte, and F. Roca (Springer, Berlin, 2012).

[2] M. Mews, M. Liebhaber, B. Rech, and L. Korte, Valence band alignment and hole transport in amorphous/crystalline silicon heterojunction solar cells, Appl. Phys. Lett. 107, 013902 (2015).

[3] J. P. Seif, D. Menda, A. Descoeudres, L. Barraud, O. Özdemir, C. Ballif, and S. De Wolf, Asymmetric band offsets in silicon heterojunction solar cells: Impact on device performance, J. Appl. Phys. 120, 054501 (2016).

[4] S. Izumi, S. Hara, T. Kumagai, and S. Sakai, Structural and mechanical properties of well-relaxed amorphous-crystal interface in silicon: Molecular dynamics study, Comput. Mater. Sci. 31, 279 (2004).

[5] C. Krzeminski, Q. Brulin, V. Cuny, E. Lecat, E. Lampin, and F. Cleri, Molecular dynamics simulation of the recrystallization of amorphous Si layers: Comprehensive study of the dependence of the recrystallization velocity on the interatomic potential, J. Appl. Phys. 101, 123506 (2007).

[6] M. Nolan, M. Legesse, and G. Fagas, Surface orientation effects in crystalline-amorphous silicon interfaces, Phys. Chem. Chem. Phys. 14, 15173 (2012).

[7] B. C. Pan and R. Biswas, Structure and simulation of hydrogenated nanocrystalline silicon, J. Appl. Phys. 96, 6247 (2004).

[8] M. Tosolini, L. Colombo, and M. Peressi, Atomic-scale model of $c$-Si/a-Si:H interfaces, Phys. Rev. B 69, 075301 (2004).

[9] I. Santos, M. Cazzaniga, G. Onida, and L. Colombo, Atomistic study of the structural and electronic properties of $a-\mathrm{Si}: \mathrm{H} / c-\mathrm{Si}$ interfaces, J. Phys. Condens. Matter 26, 095001 (2014).

[10] B. M. George, J. Behrends, A. Schnegg, T. F. Schulze, M. Fehr, L. Korte, B. Rech, K. Lips, M. Rohrmüller, E. Rauls et al., Atomic Structure of Interface States in Silicon Heterojunction Solar Cells, Phys. Rev. Lett. 110, 136803 (2013).

[11] G. Allan, C. Delerue, and M. Lannoo, Electronic structure and localized states in a model amorphous silicon, Phys. Rev. B 57, 6933 (1998).

[12] C. G. Van de Walle and L. H. Yang, Band discontinuities at heterojunctions between crystalline and amorphous silicon, J. Vac. Sci. Technol. B 13, 1635 (1995).

[13] M. Peressi, L. Colombo, and S. de Gironcoli, Role of defects in the electronic properties of amorphous/crystalline Si interface, Phys. Rev. B 64, 193303 (2001).

[14] M. Zeman and D. Zhang, Hetrojunction Silicon Based Solar Cells, in Physics and Technology of AmorphousCrystalline Heterostructure Silicon Solar Cells, edited by W. G. J. H. M. van Sark, L. Korte, and F. Roca (Springer, Berlin, 2012).

[15] G. Kresse and J. Hafner, $A b$ initio molecular dynamics for liquid metals, Phys. Rev. B 47, 558 (1993).

[16] G. Kresse and J. Furthmüller, Efficient iterative schemes for $a b$ initio total-energy calculations using a plane-wave basis set, Phys. Rev. B 54, 11169 (1996).

[17] P. E. Blöchl, Projector augmented-wave method, Phys. Rev. B 50, 17953 (1994).

[18] G. Kresse and D. Joubert, From ultrasoft pseudopotentials to the projector augmented-wave method, Phys. Rev. B 59, 1758 (1999).

[19] S. Nosé, A unified formulation of the constant temperature molecular dynamics methods, J. Chem. Phys. 81, 511 (1984). 
[20] L. E. Hintzsche, C. M. Fang, T. Watts, M. Marsman, G. Jordan, M. W. P. E. Lamers, A. W. Weeber, and G. Kresse, Density functional theory study of the structural and electronic properties of amorphous silicon nitrides: $\mathrm{Si}_{3} \mathrm{~N}_{4-x}$ :H, Phys. Rev. B 86, 235204 (2012).

[21] The average $\mathrm{Si}-\mathrm{Si}$ and $\mathrm{Si}-\mathrm{H}$ nearest-neighbor distances are very similar to those obtained in a similar-sized cell from a previous work (Ref. [22]). The largest difference of $0.02 \AA$ occurs for $\mathrm{Si}-\mathrm{H}$. The $\mathrm{Si}-\mathrm{Si}-\mathrm{Si}$ bond-angle distribution is only $0.1^{\circ}$ different, and its rms width slightly narrower $\left(11.9^{\circ}\right)$ than in the previous work $\left(12.5^{\circ}\right)$. Reference [22] used standard PAW data sets and PW91 instead of PBEsol. We compare structures that have been quenched, i.e., are at $0 \mathrm{~K}$ (the numbers listed in Ref. [22] refer to MD at $300 \mathrm{~K}$ ).

[22] K. Jarolimek, R. A. de Groot, G. A. de Wijs, and M. Zeman, First-principles study of hydrogenated amorphous silicon, Phys. Rev. B 79, 155206 (2009).

[23] J. P. Perdew, A. Ruzsinszky, G. I. Csonka, O. A. Vydrov, G. E. Scuseria, L. A. Constantin, X. Zhou, and K. Burke, Restoring the Density-Gradient Expansion for Exchange in Solids and Surfaces, Phys. Rev. Lett. 100, 136406 (2008).

[24] J. Heyd, J. E. Peralta, G. E. Scuseria, and R. L. Martin, Energy band gaps and lattice parameters evaluated with the Heyd-Scuseria-Ernzerhof screened hybrid functional, J. Chem. Phys. 123, 174101 (2005).

[25] J. Heyd, G. E. Scuseria, and M. Ernzerhof, Hybrid functionals based on a screened Coulomb potential, J. Chem. Phys. 118, 8207 (2003).

[26] J. Heyd, G. E. Scuseria, and M. Ernzerhof, Hybrid functionals based on a screened Coulomb potential, J. Chem. Phys. 118, 8207 (2003); 124, 219906(E) (2006).

[27] Z. Remeš, M. Vaněček, P. Torres, U. Kroll, A. H. Mahan, and R.S. Crandall, Optical determination of the mass density of amorphous and microcrystalline silicon layers with different hydrogen contents, J. Non-Cryst. Solids 227, 876 (1998).

[28] R. Bellisent, A. Menelle, W. S. Howells, A. C. Wright, T. M. Brunier, R. N. Sinclair, and F. Jansen, The structure of amorphous $\mathrm{Si}: \mathrm{H}$ using steady-state and pulsed neutron sources, Physica (Amsterdam) 156B, 217 (1989).

[29] S. Vignoli, P. Mélinon, B. Masenelli, P. Roca i Cabarrocas, A. M. Flank, and C. Longeaud, Over-coordination and order in hydrogenated nanostructured silicon thin films: Their influence on strain and electronic properties, J. Phys. Condens. Matter 17, 1279 (2005).

[30] M. Wakagi, K. Ogata, and A. Nakano, Structural study of $a$-Si and $a$-Si:H films by EXAFS and Raman-scattering spectroscopy, Phys. Rev. B 50, 10666 (1994).
[31] It is customary to use the average potential in the unit cell as a reference. However, any potential that has a fixed offset can be used. Here, we use the mean potential at core (see main text). We checked that the offset is rigid.

[32] S. K. O'Leary, An empirical density of states and joint density of states analysis of hydrogenated amorphous silicon: A review, J. Mater. Sci. - Mater. Electron. 15, 401 (2004).

[33] J. Tauc, R. Grigorovici, and A. Vancu, Optical properties and electronic structure of amorphous germanium, Phys. Status Solidi 15, 627 (1966).

[34] K. Jarolimek, R. A. de Groot, G. A. de Wijs, and M. Zeman, Quantum confinement and band offsets in amorphous silicon quantum wells, Phys. Rev. B 90, 125430 (2014).

[35] R. A. Street, Hydrogenated Amorphous Silicon (Cambridge University Press, Cambridge, 1991).

[36] The primitive cell is cut from the interface cell and thus the $\langle 111\rangle$ vector points along the interface normal and along the $z$ axis. This allows us to use the same Cartesian coordinates $(0.1303,0.0752,0.1064) \AA^{-1}$ for the $X$ point, in both band structure calculations.

[37] A. Baldereschi, S. Baroni, and R. Resta, Band Offsets in Lattice-Matched Heterojunctions: A Model and FirstPrinciples Calculations for GaAs/AlAs, Phys. Rev. Lett. 61, 734 (1988).

[38] The approach we follow here for $c$-Si is the standard approach for calculating heterojunction-band offsets [37]. For the $a-\mathrm{Si}: \mathrm{H}$ side of the interface the standard approach offers no apparent advantages. It requires us to determine the average potential both in the amorphous part of the interface simulation cell and for pure $a-\mathrm{Si}: \mathrm{H}$. For the latter, the band edges should be determined with a Tauc fit. Here, we circumvent the alignment step for $a$-Si:H and do the Tauc fit in the interface cell directly. This is more simple and less prone to statistical uncertainly (in determining the average potentials). For $c$-Si the "standard" approach is the preferred method, as there the average potential can be straightforwardly calculated without any statistical uncertainty.

[39] M. Liebhaber, M. Mews, T. F. Schulze, L. Korte, B. Rech, and K. Lips, Valence band offset in heterojunctions between crystalline silicon and amorphous silicon (sub)oxides $\left(a-\mathrm{SiO}_{x}: \mathrm{H}, 0<x<2\right)$, Appl. Phys. Lett. 106, 031601 (2015).

[40] L. Shen, F. Meng, and Z. Liu, Roles of the Fermi level of doped $a-\mathrm{Si}: \mathrm{H}$ and band offsets at $a-\mathrm{Si}: \mathrm{H} / c$-Si interfaces in n-type HIT solar cells, Solar Energy 97, 168 (2013). 